\title{
The Honeybee Antimicrobial Peptide Apidaecin Differentially Immunomodulates Human Macrophages, Monocytes and Dendritic Cells
}

\author{
Regina Tavano $^{a, b}$ Daniela Segat ${ }^{a, b}$ Marina Gobbo ${ }^{c}$ Emanuele Papini ${ }^{a, b}$ \\ ${ }^{a}$ Centro di Ricerca Interdipartimentale per le Biotecnologie Innovative, ${ }^{b}$ Dipartimento di Scienze Biomediche \\ Sperimentali, and ${ }^{\mathrm{C} D i p a r t i m e n t o ~ d i ~ S c i e n z e ~ C h i m i c h e, ~ U n i v e r s i t a ̀ ~ d i ~ P a d o v a, ~ P a d o v a, ~ I t a l y ~}$
}

Key Words
Antimicrobial peptides $\cdot$ Apidaecin $\cdot$ Chemokines
Cytokines $\cdot$ Honeybee $\cdot$ Macrophages $\cdot$ Monocytes

\begin{abstract}
We show that apidaecin binds to human macrophages, monocytes and dendritic cells, displaying different intracellular distributions and inducing diversified effects. An apidaecin-cell association was detectable at concentrations as low as $5 \mu \mathrm{M}$ and increased without saturation until $60 \mu \mathrm{M}$, was receptor independent and required a physiological temperature $\left(37^{\circ} \mathrm{C}\right)$. For apidaecin, cytosolic localization was prevalent in macrophages and endosomal localization in monocytes, and associations with the plasma membrane were predominant in dendritic cells. Apidaecin upregulated T-lymphocyte co-stimulatory molecule CD80 and cytokine/ chemokine production in macrophages, but not in monocytes and dendritic cells. Suboptimal stimulatory doses (5$10 \mu \mathrm{M})$ of apidaecin partially inhibited the lipopolysaccharide (LPS)-induced increase in major histocompatibility complex class II (MHCII) and CD86 in macrophages, and the release of selected cytokines/chemokines by both macrophages [interleukin (IL)- 6 and tumor necrosis factor (TNF)- $\alpha$ ] and monocytes [IL-6, TNF- $\alpha$, basic fibroblast growth factor (FGF) and eotaxin]. Apidaecin had a double-edged effect: at
\end{abstract}

low concentrations it partially antagonized LPS-stimulatory effects on both macrophages and monocytes while it stimulated pro-inflammatory and pro-immune functions of macrophages at higher concentrations.

Copyright $\odot 2011$ S. Karger AG, Basel

\section{Introduction}

A variety of innate antimicrobial peptides (AMPs) are distributed in living beings [1]. Membrane-active AMPs, such as defensins, perturb the bacterium plasma membrane, while non-lytic ones, like insect apidaecins, translocate into the microorganism cytosol [2]. Several AMPs may also regulate innate and adaptive immune cell functions [3-5], such as chemotaxis, apoptosis, cytokine/chemokine production $[6,7]$, antigen presentation and Th1/ Th2 balance $[6,8]$.

Insect hemolymph contains AMPs ensuring protection against bacterial infections. Among these, the prorich 18-20 residues of apidaecins are potentially exploitable in medical and biotechnology applications as novel antibiotic drugs [9], being poorly structured, devoid of S-S bridges, easily synthesizable and not toxic to human cells [10]. Apidaecin, like similar pro-rich peptides (drosocin, pyrrhocoricin and Bac7), acts via a three-step pro-

\section{KARGER \\ Fax +4161306 1234 \\ E-Mail karger@karger.ch}

www.karger.com (c) 2011 S. Karger AG, Basel

1662-811X/11/0036-0614\$38.00/0

Accessible online at:

www.karger.com/jin
Dr. Regina Tavano

Dipartimento di Scienze Biomediche Sperimentali, Università di Padova

Via U. Bassi 58/B

IT-35131 Padova (Italy)

Tel. +39049827 6478, E-Mail regina.tavano@unipd.it 
cess involving: (i) bacterial surface binding, (ii) translocation into the cytoplasm and (iii) binding to DnaK, the Escherichia coli homologue of human HSP70 [2, 11-12]. While the bacteriostatic action of apidaecin has been extensively studied [13-15], its immunostimulatory activity has not been elucidated yet. Based on the structural similarity between mammalian and insect pro-rich AMPs, we hypothesized that apidaecin, although produced by phylogenetically distant organisms, can cross talk with the human immune system. This possibility is supported by the observation that pro-rich mammalian peptides Bac7 and PR39 can interact with immune cells [16-18]. We show here for the first time that apidaecin associates without inducing cytotoxic effects to three important human immune cells: macrophages, monocytes and dendritic cells. Interestingly, we found that apidaecin effectively translocates into the cell cytosol of macrophages, but remains mostly endosomal or plasma membrane associated in monocytes and dendritic cells, respectively. Consistently, we show that apidaecin differentially regulates their basal and lipopolysaccharide (LPS)-induced cytokine/chemokine release and the expression of proteins necessary for antigen presentation and T-lymphocyte activation, showing a higher effect on macrophages. Our data suggest that apidaecin is partially anti-inflammatory in the presence of microbial stimuli and has a proimmune/pro-inflammatory action when acting alone.

\section{Materials and Methods}

\section{Synthesis of Apidaecin and Its Derivatives}

Full-length apidaecin Ib (GNNRPVYIPQPRPPHPRL), the peptide sequence corresponding to amino acids 13-18 of apidaecin and their fluoresceinated analogues were synthesized by solid-phase synthesis as previously reported [10]. Following the same protocol, an apidaecin derivative extended at the $\mathrm{N}$-terminus with a cysteine residue (Cys-apidaecin) was prepared and used after HPLC purification in affinity chromatography. The product was characterized by electrospray ionization mass spectrometry (MARINER API-TOF workstation), and its purity (>95\%) was confirmed by analytical HPLC, performed on a Dionex Summit Dual Gradient apparatus equipped with a Vydac C18 column $(250 \times 4.6 \mathrm{~mm}, 5 \mu \mathrm{m})$.

\section{Preparation of Human Monocytes, Macrophages and}

Dendritic Cells

Monocyte-enriched preparations were isolated from buffy coats of blood by centrifugation over a Ficoll-Hypaque (Amersham Biosciences) step gradient and a subsequent Percoll (Amersham Biosciences) gradient as described [19]. Unless otherwise specified, cells were kept at $37^{\circ} \mathrm{C}$ in a humidified atmosphere containing 5\% (v/v) $\mathrm{CO}_{2}$ in RPMI-1640 supplemented with 10\% FCS. Human macrophages were derived from monocytes cultured for
7 days with $100 \mathrm{ng} / \mathrm{ml}$ macrophage colony-stimulating factor (MCSF; PeproTech) in RPMI-1640 supplemented with 20\% FCS. On day 4, M-CSF was added again. Human dendritic cells were obtained as described [20]: following this procedure $>90 \%$ cells belonged to the immature dendritic cell phenotype.

\section{Cytofluorimetric Analysis}

The day before the experiment, human monocytes/macrophages or dendritic cells were incubated for different times with apidaecin (5-50 $\mu \mathrm{M})$ with or without LPS $0.05-0.2 \mu \mathrm{g} / \mathrm{ml}$ (Sigma). The cells were then incubated at 37 or $0^{\circ} \mathrm{C}$, depending on the experiment, in RPMI medium supplemented with $10 \%$ FCS. Supernatants were collected and cells were then washed with PBS, resuspended in FACS buffer (PBS, 1\% FBS) and 10,000 events/ sample were analyzed using a FACSCanto ${ }^{\circledR}$ analyzer (Becton Dickinson). Dead cells were excluded from FACS analysis by adding propidium iodide. Data were processed using the FACSDiva software. For the analysis of surface marker expression, after the incubation, cells were washed, resuspended in FACS buffer, divided in different samples and incubated with anti-major histocompatibility complex class II (MHCII), CD80, CD86 and ICAM1-phycoerythrin labelled antibodies (Biolegend) for $30 \mathrm{~min}$ at $4^{\circ} \mathrm{C}$. Cells were then washed, resuspended in FACS buffer and analyzed by cytofluorimetry.

\section{Confocal Analysis}

Macrophages, monocytes or dendritic cells $\left(2 \times 10^{6}\right.$ cells/ well) were seeded on cover glasses and incubated for $18 \mathrm{~h}$ at $37^{\circ} \mathrm{C}$ with fluoresceinated apidaecin. Unfixed cells were briefly rinsed with PBS and directly analyzed under a confocal microscope (SP2 Leica). As a control, cells without apidaecin have been used. Alternatively, adherent macrophages were incubated for $18 \mathrm{~h}$ with $30 \mu \mathrm{M}$ apidaecin at $37^{\circ} \mathrm{C}$; after three washes with PBS, cells were fixed in cold methanol for $10 \mathrm{~min}$ and permeabilized with cold acetone for $10 \mathrm{~min}$. Before addition of primary antibodies, immunoglobulin binding of monocytes was saturated by treatment with pooled human IgG (Sigma Chemical Co., $10 \mathrm{mg} / \mathrm{ml}$ in PBS). Cells were incubated overnight at $4^{\circ} \mathrm{C}$ with rabbit polyclonal antiapidaecin antibody, washed three times and further incubated for 40 min with secondary FITC-labelled anti-rabbit antibody (Calbiochem). After three washes, coverslips were mounted in mounting medium (KPL) and were analyzed with a confocal microscope (SP2 Leica). Images have been acquired with the different fluorescence filters; representative pictures were collected as Tiff files and processed with standard imaging programs.

\section{Bio-Plex Multiplex Cytokine Assays}

A Bio-Plex assay was performed as previously described [19]. The fluorescence intensity of the beads was measured with a Luminex system (Bio-Rad) and data were analyzed with Bio-Plex Manager software.

\section{Peptide-Carrier Conjugation}

Cys-apidaecin (2 mg) was conjugated with $2 \mathrm{mg}$ of mcKLH (Pierce) in conjugation buffer, following the manufacturer's instructions. The conjugate was subsequently purified by gel filtration using Sephadex G-25 resin (Sigma). Fractions containing proteins were mixed, divided in aliquots and frozen in liquid nitrogen. 


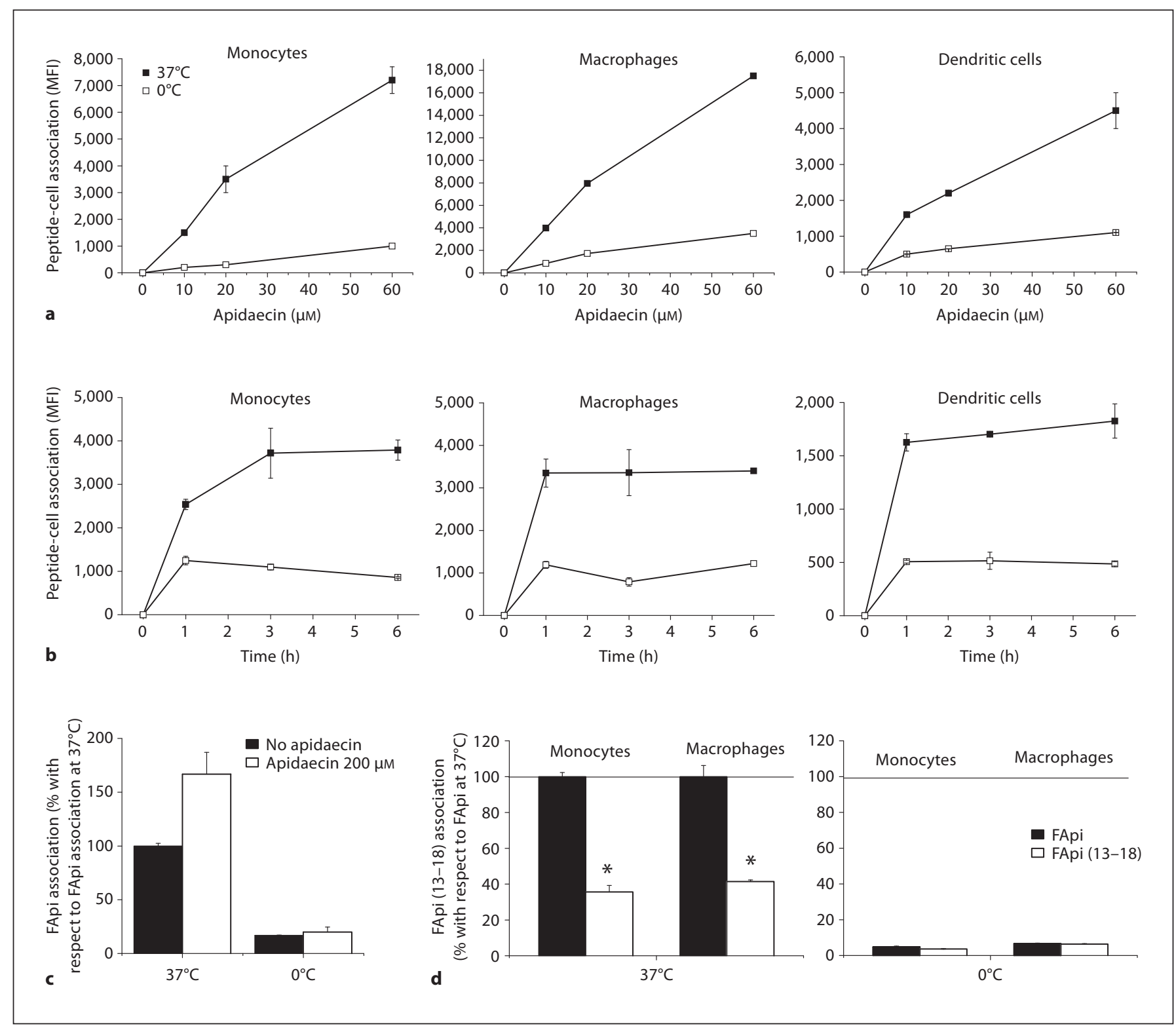

Fig. 1. Dose (a)- and time-dependent uptake of apidaecin (b) in human monocytes, macrophages and dendritic cells. Cells were incubated at 37 or $0^{\circ} \mathrm{C}$ for $1 \mathrm{~h}$ with 10,20 or $60 \mu \mathrm{M}$ (a) or for 1,3 or $6 \mathrm{~h}$ with $10 \mu \mathrm{M}$ (b) of fluoresceinated apidaecin (FApi). Cells were then washed with PBS, trypsinized and resuspended in FACS buffer. Mean fluorescence intensity (MFI) of at least 10,000 cells/sample due to FITC-conjugated peptide uptake was quantified. Data are from one of two representative experiments, run in triplicate, and bars represent ranges. c Competition assay. Human monocytes were incubated with $5 \mu \mathrm{M}$ FApi at 37 or $0^{\circ} \mathrm{C}$ for $3 \mathrm{~h}$ with or without $200 \mu \mathrm{M}$ non-labelled apidaecin. Cells were then washed, trypsinized and analyzed by FACS. MFI values are the mean of three experiments run in duplicate, and are expressed as percent of the signal produced by labelled peptide alone at $37^{\circ} \mathrm{C}$. Means \pm SE. d Different uptake of the whole peptide and the conserved region of apidaecin (residues 13-18). Human monocytes or macrophages were incubated with $10 \mu \mathrm{M}$ of FApi or FApi (13-18) for $3 \mathrm{~h}$ at 37 or $0^{\circ} \mathrm{C}$. Cells were then washed with PBS, trypsinized and resuspended in FACS buffer. MFI due to FITC-conjugated peptides was quantified. Data are from one of two representative experiments run in triplicate, and percentage values were calculated considering 100 for FApi association at $37^{\circ} \mathrm{C}$ in monocytes and macrophages, respectively. Means $\pm \mathrm{SE} .{ }^{*} \mathrm{p} \leq 0.05$. 
Immunization and Production of Polyclonal Antibodies

Male adult New Zealand white rabbits (Harlan, Italy) were immunized by subcutaneous injection with an aliquot $(750 \mu \mathrm{l})$ of carrier-peptide conjugate mixed 1:1 v/v with complete Freund's adjuvant (Sigma). Three subsequent injections were administered at 14-day intervals with incomplete Freund's adjuvant (Sigma). Anti-sera were taken on day 68 and antibodies were purified by affinity chromatography on apidaecin-modified columns. Briefly, columns were prepared with $2.5 \mathrm{ml}$ of $50 \%$ SulfoLink coupling gel (Pierce) and $2 \mathrm{mg}$ of Cys-apidaecin dissolved in coupling buffer (50 mM Tris-HCl, 5 mM EDTA, pH 8.5). Columns were then washed with coupling buffer and the non-specific binding sites were blocked with a solution of $50 \mathrm{~mm}$ cysteine (Sigma) for $30 \mathrm{~min}$ at room temperature. Subsequently, columns were washed again extensively, and antisera (diluted 1:1 v/v with PBS) were added to the columns; after extensive washing, antibodies were eluted with $0.2 \mathrm{M}$ glycine, $\mathrm{pH} 2.8$.

\section{Measurement of the LPS-Binding Activities of Apidaecin}

The assay was performed as described [21]. Briefly, the day before the experiment, NUNC plates were incubated with $100 \mu \mathrm{l}$ of PBS with or without $1 \mu \mathrm{g}$ of LPS (Sigma)/well, at room temperature. The day of the experiment, after some washing, wells were saturated for $1 \mathrm{~h}$ at room temperature with $1 \%$ BSA in PBS. Then the plate was incubated for $1 \mathrm{~h} 30 \mathrm{~min}$ at $37^{\circ} \mathrm{C}$ with different peptide concentrations. After some washing, $100 \mathrm{ng}$ of anti-apidaecin were added to each well and incubated for $90 \mathrm{~min}$ at room temperature. Then $100 \mu$ l of 1/200 anti-rabbit HRP (Calbiochem) were added and incubated for $1 \mathrm{~h}$ at room temperature. Finally, the peroxidase substrate solution (ABTS) was added to each well. Optical density was assessed at $405 \mathrm{~nm}$ on an automatic microplate reader (Amersham).

\section{Statistical Analysis}

Results were expressed as means \pm SEM. Significance ( $\mathrm{p} \leq$ 0.05 ) of differences with respect to control values were calculated by two-population $t$ tests.

\section{Results}

Flow-cytofluorimetric experiments with a fluorescent-labelled analogue showed that the interaction between apidaecin and macrophages, monocytes and dendritic cells is comparable, although efficacy is slightly decreasing (fig. 1a, b). At $37^{\circ} \mathrm{C}$, apidaecin cell uptake reached an equilibrium after $\sim 1-3 \mathrm{~h}$ and was hardly saturable at concentrations of up to $60 \mu \mathrm{M}(150 \mu \mathrm{g} / \mathrm{ml})$. At $0^{\circ} \mathrm{C}$, apidaecin-cell association was markedly reduced. Competition assays (fig. 1c) proved that this process does not require high-affinity receptors, similarly to what was observed in epithelial cells [10]. Cell association with a fluorescent, shorter peptide corresponding to the C-terminal sequence $13-18$, conserved in different apidaecin isoforms, was significantly decreased at $37^{\circ} \mathrm{C}$, but comparable to that of full-length apidaecin at $0^{\circ} \mathrm{C}$, suggesting

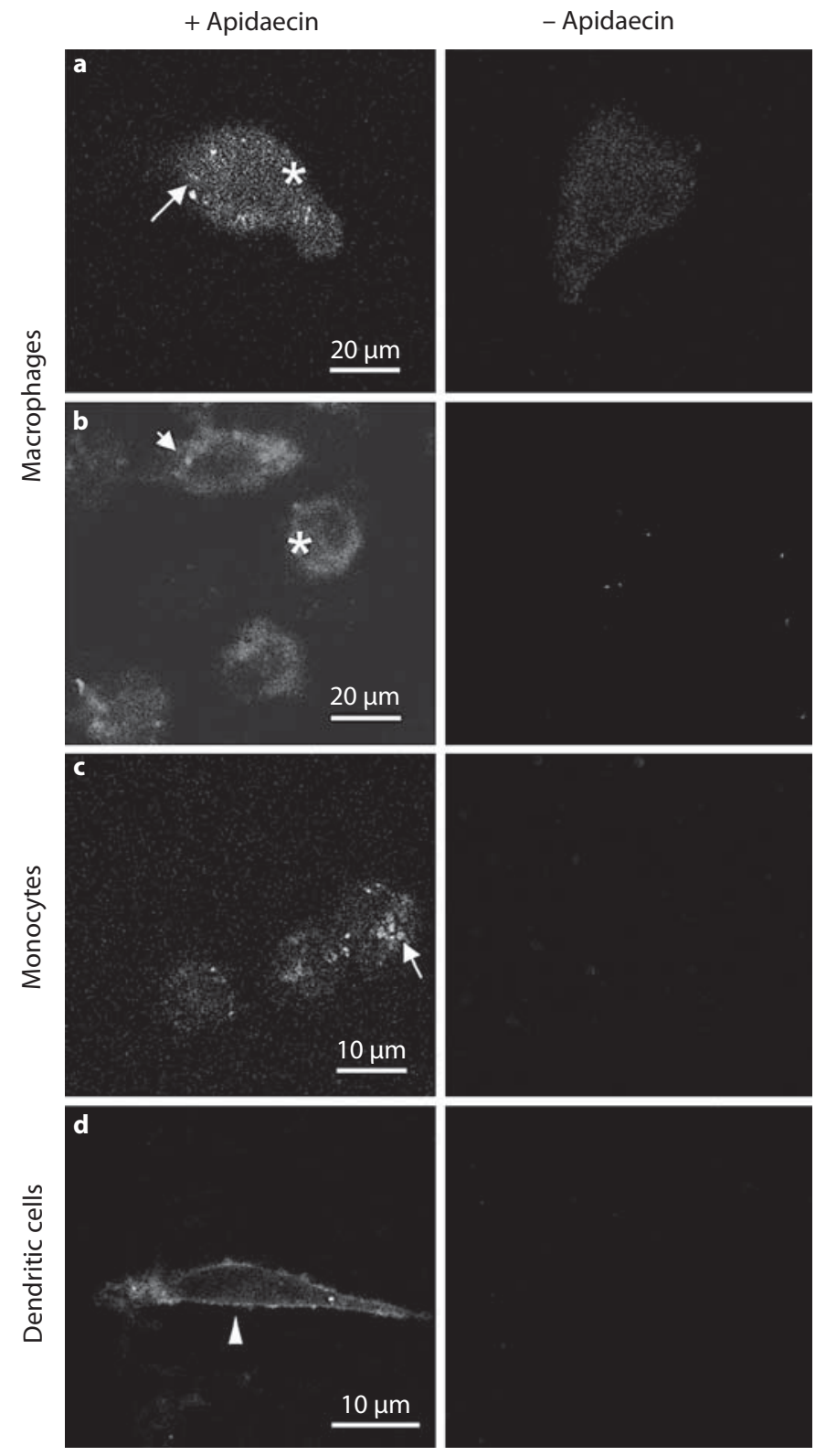

Fig. 2. Confocal analysis of human monocytes, macrophages or dendritic cells treated with apidaecin. Cells grown on glass coverslips were incubated with $30 \mu \mathrm{M}$ apidaecin, fixed with methanol, and permeabilized with acetone; cells were then subjected to indirect immunostaining with specific anti-apidaecin rabbit antibodies and with FITC-labelled anti-rabbit antibodies and analyzed by confocal microscopy (a). Alternatively, cells adherent to glass coverslips were incubated for $18 \mathrm{~h}$ with culture medium containing $30 \mu \mathrm{M}$ fluoresceinated apidaecin at $37^{\circ} \mathrm{C}$, briefly rinsed in PBS and then directly analyzed using a confocal microscope (b-d). Control cells were treated with no apidaecin. Arrowhead indicates the plasma membrane, arrows intracellular vesicles, and asterisks indicate the cytosol. 


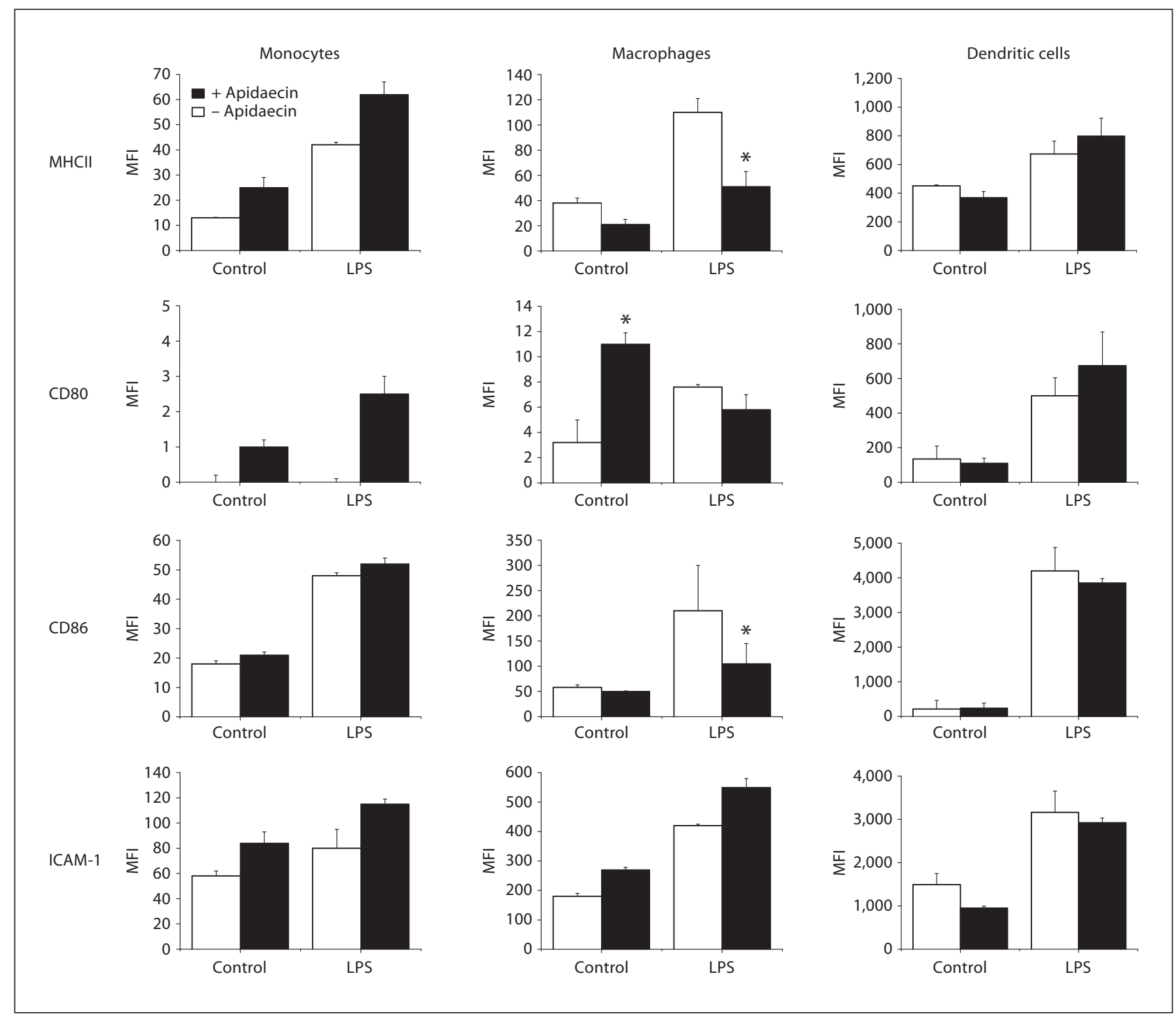

Fig. 3. Effect of apidaecin on CD80, CD86, MHCII and ICAM-1 expression in monocytes, macrophages and dendritic cells. Adherent human monocytes, differentiated macrophages or dendritic cells were cultured in RPMI plus $10 \%$ FCS with $10 \mu \mathrm{M}$ apidaecin and $0.2 \mu \mathrm{g} / \mathrm{ml}$ E. coli LPS, as indicated. After $24 \mathrm{~h}$, cells

that the lacking $\mathrm{N}$-terminal sequence is necessary for efficient cell capture of apidaecin at physiological temperature (fig. 1d). Cell binding was not enhanced by the absence of serum in the extracellular medium, suggesting that serum $(10 \% \mathrm{v} / \mathrm{v})$ does not interfere with peptide cell association (data not shown).

The intracellular distribution of apidaecin in the three cell types was analyzed by fluorescence confocal micros- were collected and the indicated markers were stained with phycoerythrin-labelled anti-CD-specific antibodies and analyzed by FACS. Data are expressed as MFI and are the mean \pm SE of four experiments run in triplicate. ${ }^{*} \mathrm{p} \leq 0.05 \mathrm{vs}$. control (no stimulus or LPS stimulated only).

copy (fig. 2). Pictures showed significant differences: in monocytes, the bee peptide distributes in a punctuated way, which is compatible with endocytic vesicles, but in a more diffuse way in macrophages, which is compatible with cytosolic translocation. In contrast, in dendritic cells, apidaecin is apparently mainly restricted to the plasma membrane and is found in reduced quantity in intracellular compartments. 


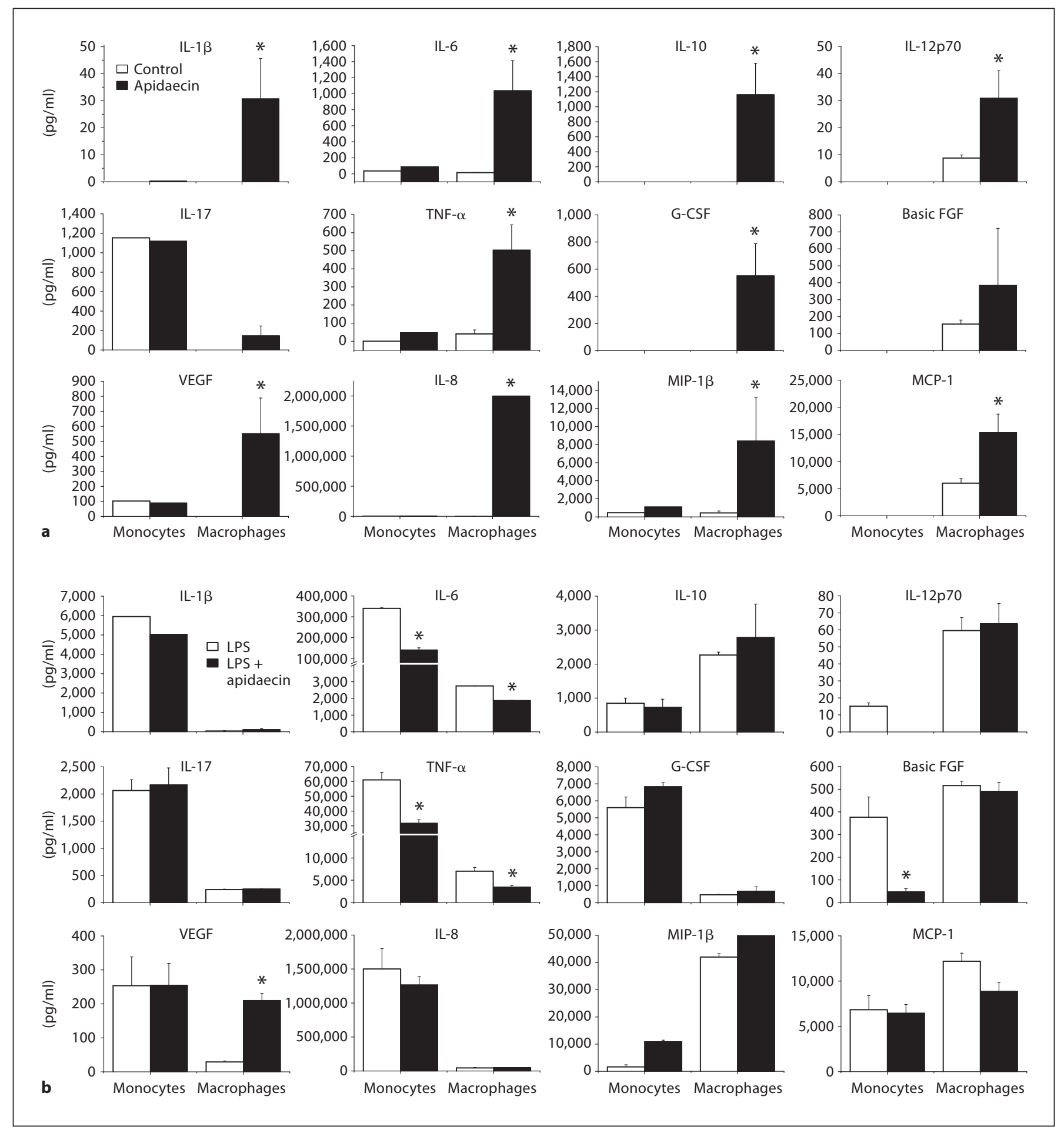

Fig. 4. Effect of apidaecin on cytokine/chemokine expression in monocytes and macrophages stimulated with or without LPS. Adherent human monocytes or differentiated macrophages were cultured in RPMI medium plus 10\% FCS in the presence of apidaecin $(10 \mu \mathrm{M})$ or with no agonist (a). Alternatively, cells were treated with LPS $(0.2 \mu \mathrm{g} / \mathrm{ml})$ or with LPS and apidaecin $(10 \mu \mathrm{M}$; b). After 24-hour stimulation, culture media were collected and analyzed by a 23 -analyte Bio-Plex assay. Values $(\mathrm{pg} / \mathrm{ml})$ are the mean from three experiments run in duplicate \pm SE and only refer to analytes which were detectable in the assays. ${ }^{*} p \leq 0.05$ vs. cells treated with no stimulus (a) or with LPS alone (b). 
Fig. 5. Dose-response curves of the effect of apidaecin on IL- 6 and TNF- $\alpha$ production in human monocytes and macrophages. Values are the mean from three experiments run in duplicate \pm SE. a Cells were incubated for $18 \mathrm{~h}$ with different concentrations of apidaecin (5-50 $\mu \mathrm{M})$. IL-6 and TNF- $\alpha$ concentrations were determined in supernatants by ELISA. b Human macrophages were stimulated with different concentrations of apidaecin (5$50 \mu \mathrm{M})$ in the presence of $0.05 \mu \mathrm{g} / \mathrm{ml}$ of LPS. IL- 6 and TNF- $\alpha$ concentrations in the supernatants were calculated by ELISA. ${ }^{*} \mathrm{p} \leq 0.05$ vs. cells treated with LPS alone.

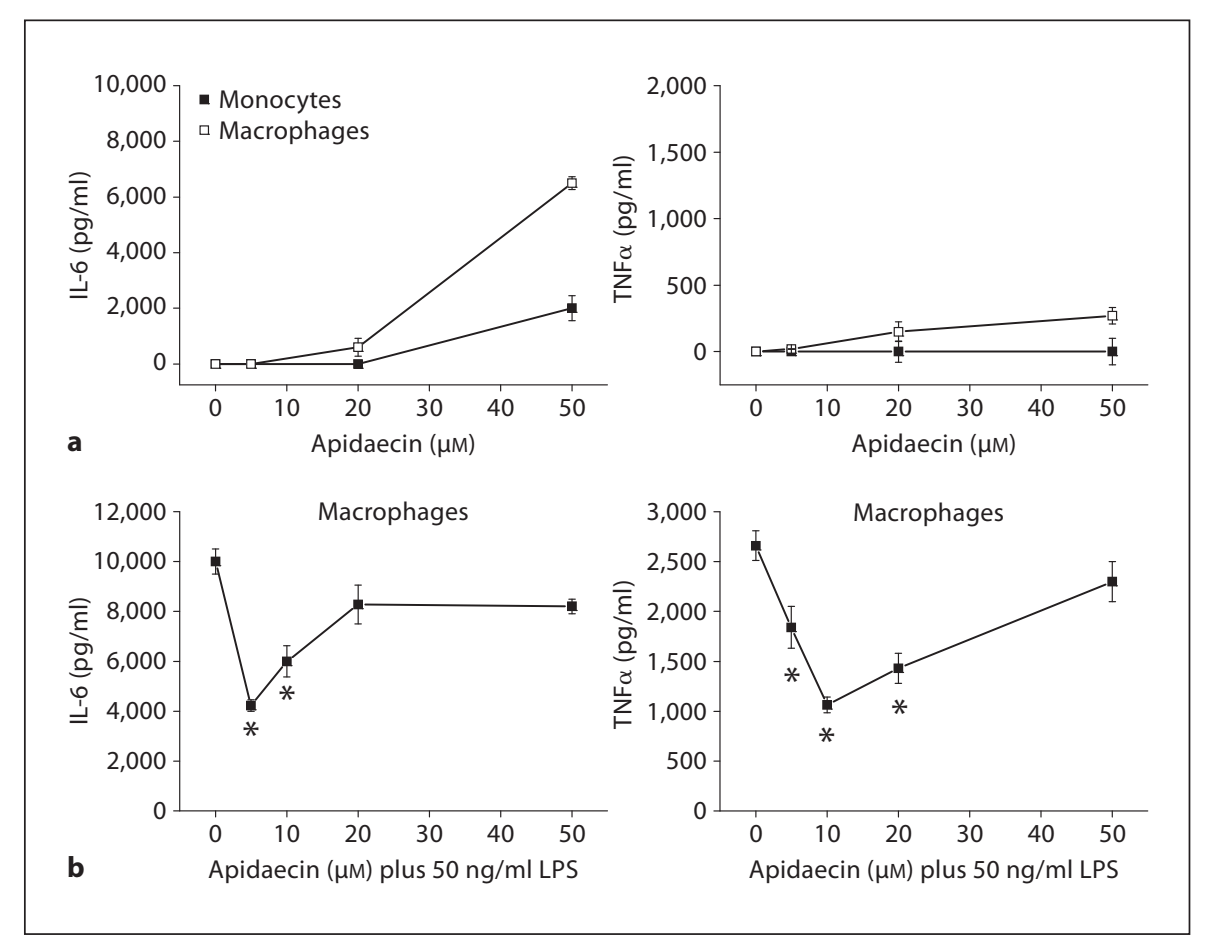

The effects of apidaecin on the basal or LPS-induced expression of MHCII, CD80, CD86 and ICAM-1 were analyzed by flow-cytofluorimetry. Figure 3 shows that macrophage CD80 expression was upregulated by the peptide, reaching a value similar to that induced by LPS, while monocytes and dendritic cells were not influenced. On the contrary, endotoxin-induced upregulation of CD86 and MHCII was significantly reduced in macrophages ( $\sim 70 \%$ inhibition). Again LPS-induced markers were not affected in monocytes and dendritic cells. Plasma membrane damage, tested by propidium iodide exclusion evaluation run in parallel, was never increased by apidaecin (not shown).

Suspension array assays (Bio-Plex) proved that apidaecin stimulated a significantly higher release of interleukin (IL)-1 $\beta$, IL-6, IL-10, IL-12p70, tumor necrosis factor (TNF)- $\alpha$, granulocyte colony-stimulating factor (G$\mathrm{CSF}$ ), basic fibroblast growth factor (FGF), vascular endothelial growth factor (VEGF), IL-8, macrophage inflammatory protein (MIP) $1 \beta$ and macrophage chemotactic peptide (MCP)-1 compared to control cells (fig. 4) in macrophages only. Apidaecin $(10 \mu \mathrm{M})$ determined a partial but significant reduction in LPS-induced secretion of IL- 6 and TNF- $\alpha$ in both monocytes (-60 and $-50 \%$, respectively) and macrophages $(-40$ and $-60 \%$, respectively), with a more marked decrease in basic FGF and eotaxin secretion in monocytes only ( -85 and $-90 \%$, respectively). The release of other cytokines/chemokines remained unaffected. Apidaecin induced neither cytokine/chemokine production in dendritic cells nor LPSinduced effects (not shown).

Dose-response analyses of apidaecin-dependent induction of cytokines and inhibition of LPS-dependent effects are shown in figure 5. Apidaecin stimulated macrophages to secrete IL- 6 and TNF- $\alpha$ more efficiently than monocytes at all concentrations tested. The peptide effect was not significant at concentrations $<5 \mu \mathrm{M}$ but increased steadily at higher concentrations. Interestingly, the antiLPS action of apidaecin is maximal at around 5-10 $\mu \mathrm{M}$ but reverts at higher concentrations, presumably for the concomitant induction of peptide-dependent cytokine stimulation.

To gain more insight into the mechanism of the modulation of apidaecin-LPS effects, we determined whether it could directly bind to LPS, thus inhibiting its interaction with CD14, the receptor expressed on the monocyte/ macrophage surface. Literature data are conflicting on this point: in fact Otvos et al. [11] demonstrated that apidaecin could bind to LPS, while Dutta et al. [14] showed that apidaecin-LPS binding was weak or absent. To address this issue we performed an ELISA: results demonstrated that apidaecin-LPS binding was not strong and 
specific, suggesting that the anti-endotoxic activity of the bee peptide is not due to the interaction between the peptide and LPS (not shown).

\section{Discussion}

In this study, we document for the first time that apidaecin associates with human macrophages, monocytes and dendritic cells with no need of specific membrane receptors, extending our previous observations that this bee AMP can cross the plasma membrane of epithelial eukaryotic cells, without determining cytotoxic effects [10]. A physiological temperature $\left(37^{\circ} \mathrm{C}\right)$ is required for optimal cell binding, suggesting that either membrane fluidity or cooperation of protein carriers with low affinity and high capacity are necessary for optimal cell interaction. This process appears, at least in part, specific, since the removal of the 1-12 N-terminal sequence determined a reduction in apidaecin cell association. Confocal microscopy suggests that part of apidaecin can translocate into the cell cytosol after plasma membrane binding. However, cytosolic translocation is efficient in macrophages but less evident in monocytes and dendritic cells. In fact, in monocytes the peptide prevailed in structures resembling endolysosomal compartments, while in dendritic cells the peptide remains more associated with cell plasma. This cell-specific cellular localization also strongly suggests that apidaecin cell translocation into the cytosol is a specific process, and further studies are necessary to elucidate its molecular mechanism. From the functional view point, we show for the first time that apidaecin has an immunomodulating effect in human cells. Macrophages, monocytes and dendritic cells, although similarly associating with apidaecin, were differentially sensitive to the peptide. Macrophages were mostly influenced: CD80, the Th-lymphocyte co-stimulatory protein, was upregulated by apidaecin. On the contrary, we did not observe a similar action on monocytes and dendritic cells. Analysis of the modulation of LPS-induced effects on our cell panel confirmed the macrophage-selective effect of apidaecin on the expression of activation markers. In this case apidaecin partially opposed the increase in MHCII and CD86 expression induced by LPS. Again, such an anti-LPS action was absent in dendritic cells and monocytes, confirming the propensity of apidaecin to be macrophage selective. The differential action of apidaecin on the LPS stimulus suggests that the bee peptide does not merely complex with LPS thus diminishing its action, because in this case dendrit-

Apidaecin Modulates the Human

Immune System ic cells and monocytes would have been subjected to the same inhibition of LPS effects on CDs. Indeed, we could scarcely find a binding between apidaecin and LPS.

Consistently with CD80 overexpression, we found that apidaecin-treated macrophages increased their release of many different cytokines and chemokines, while monocytes and dendritic cells remained unaltered. Again, apidaecin partially inhibited IL- 6 and TNF- $\alpha$ release induced by LPS, but in this case not only macrophages but also monocytes were affected. Interestingly, at low peptide concentrations the partial LPS-inhibitory effect is predominately restricted to the ability to stimulate cytokines, while, on the contrary, at higher concentrations its intrinsic ability to induce cytokine stimulation annihilates the anti-LPS effects.

Our data suggest that apidaecin could render human macrophages more efficient in their antimicrobial action due to an increased exposure of T-lymphocyte co-stimulatory molecules and the release of several cytokines/chemokines. On the contrary, the lack of apidaecin effects on circulating monocytes would prevent dangerous systemic effects, e.g. anaphylactic or septic shock. The inability of apidaecin to stimulate the immune functions of dendritic cells suggests that it is devoid of pro-adjuvant properties.

Although our data do not demonstrate the biochemical target of apidaecin in the immune cells, they show a correlation between the intensity of the biological activity, displayed in macrophages, and the cytosolic translocation. This suggests that the interaction with one or more intracellular targets mediates the cell action of apidaecin.

In conclusion, we suggest that apidaecin at doses of 5-10 $\mu \mathrm{M}$ could improve the antimicrobial effects of macrophages via increased antigen presentation and cytokine release. It also appears that activation of macrophages as well as monocytes by strong pro-inflammatory pathogen-associated molecular patterns, e.g. Gram-negative LPS, is modulated in the presence of low concentrations $(5-10 \mu \mathrm{M})$ of apidaecin, suggesting that apidaecin may also acts as a mild anti-inflammatory agent, consistent with inhibition of NF- $\kappa \mathrm{B}$-dependent expression of adhesion molecules and ischemia-reperfusion injury by mammalian analogue of apidaecin (PR 39) in a rat model [22, 23].

Our in vitro observations have to be confirmed in vivo in future experiments to assess if the peculiar immunomodulatory activity of bee peptides may be useful for the development and refinement of natural immune-potentiating or anti-inflammatory drugs.

J Innate Immun 2011;3:614-622 


\section{Acknowledgment}

We thank the Centro Trasfusionale of the Hospital of Padua (ULSS 16) for providing buffy coats.

\section{Disclosure Statement}

This research was supported by a grant from the University of Padova (Ex 60\%, 2010). The authors have no other relevant affiliations or financial involvement with any organization or entity with a financial interest in or financial conflict with the subject or material discussed.

\section{References}

1 Mookherjee N, Hancock RE: Cationic host defence peptides: innate immune regulatory peptides as a novel approach for treating infections. Cell Mol Life Sci 2007;64:922-933.

$>2$ Castle M, Nazarian A, Yi SS, Tempst P: Lethal effects of apidaecin on Escherichia coli involve sequential molecular interactions with diverse targets. J Biol Chem 1999;274: 32555-32564.

3 Selsted ME, Ouellette AJ: Mammalian defensins in the antimicrobial immune response. Nat Immunol 2005;6:551-557.

-4 Guani-Guerra E, Santos-Mendoza T, LugoReyes SO, Teran LM: Antimicrobial peptides: general overview and clinical implications in human health and disease. Clin Immunol 2010;135:1-11.

5 Yang D, Biragyn A, Hoover DM, Lubkowski J, Oppenheim JJ: Multiple roles of antimicrobial defensins, cathelicidins, and eosinophilderived neurotoxin in host defense. Annu Rev Immunol 2004;22:181-215.

$\checkmark 6$ Brown KL, Hancock RE: Cationic host defense (antimicrobial) peptides. Curr Opin Immunol 2006;18:24-30.

7 Mookherjee N, Lippert DN, Hamill P, Falsafi R, Nijnik A, Kindrachuk J, Pistolic J, Gardy J, Miri P, Naseer M, Foster LJ, Hancock RE: Intracellular receptor for human host defense peptide LL-37 in monocytes. J Immunol 2009; 183:2688-2696.

$>8$ Kurosaka K, Chen Q, Yarovinsky F, Oppenheim JJ, Yang D: Mouse cathelin-related antimicrobial peptide chemoattracts leukocytes using formyl peptide receptor-like 1/ mouse formyl peptide receptor-like 2 as the receptor and acts as an immune adjuvant. J Immunol 2005;174:6257-6265. $\checkmark 9$ Li WF, Ma GX, Zhou XX: Apidaecin-type peptides: biodiversity, structure-function relationships and mode of action. Peptides 2006;27:2350-2359.

10 Gobbo M, Benincasa M, Bertoloni G, Biondi B, Dosselli R, Papini E, Reddi E, Rocchi R, Tavano R, Gennaro R: Substitution of the arginine/leucine residues in apidaecin Ib with peptoid residues: effect on antimicrobial activity, cellular uptake, and proteolytic degradation. J Med Chem 2009;52:5197-5206.

- 11 Otvos L Jr, O I, Rogers ME, Consolvo PJ, Condie BA, Lovas S, Bulet P, BlaszczykThurin M: Interaction between heat shock proteins and antimicrobial peptides. Biochemistry 2000;39:14150-14159.

$\checkmark 12$ Scocchi M, Lüthy C, Decarli P, Mignogna G, Christen P, Gennaro R: The proline-rich antibacterial peptide Bac7 binds to and inhibits in vitro the molecular chaperone DnaK. Int J Pept Res Ther 2009;15:147.

13 Zhou XX, Li WF, Pan YJ: Functional and structural characterization of apidaecin and its N-terminal and C-terminal fragments. J Pept Sci 2008;14:697-707.

14 Dutta RC, Nagpal S, Salunke DM: Functional mapping of apidaecin through secondary structure correlation. Int J Biochem Cell Biol 2008;40:1005-1015.

15 Taguchi S, Mita K, Ichinohe K, Hashimoto S: Targeted engineering of the antibacterial peptide apidaecin, based on an in vivo monitoring assay system. Appl Environ Microbiol 2009;75:1460-1464.

-16 Sadler K, Eom KD, Yang JL, Dimitrova Y, Tam JP: Translocating proline-rich peptides from the antimicrobial peptide bactenecin 7 . Biochemistry 2002;41:14150-14157.

-17 Tomasinsig L, Skerlavaj B, Papo N, Giabbai B, Shai Y, Zanetti M: Mechanistic and functional studies of the interaction of a prolinerich antimicrobial peptide with mammalian cells. J Biol Chem 2006;281:383-391.
18 Catrina SB, Refai E, Andersson M: The cytotoxic effects of the anti-bacterial peptides on leukocytes. J Pept Sci 2009;15:842-848.

19 Tavano R, Franzoso S, Cecchini P, Cartocci E, Oriente F, Arico B, Papini E: The membrane expression of Neisseria meningitidis adhesin $\mathrm{A}(\mathrm{Nad} \mathrm{A})$ increases the proimmune effects of MenB OMVs on human macrophages, compared with NadA-OMVs, without further stimulating their proinflammatory activity on circulating monocytes. J Leukoc Biol 2009;86:143-153.

20 Sallusto F, Lanzavecchia A: Efficient presentation of soluble antigen by cultured human dendritic cells is maintained by granulocyte/ macrophage colony-stimulating factor plus interleukin 4 and downregulated by tumor necrosis factor alpha. J Exp Med 1994;179: 1109-1118.

21 Nagaoka I, Hirota S, Niyonsaba F, Hirata M, Adachi Y, Tamura H, Heumann D: Cathelicidin family of antibacterial peptides CAP18 and CAP11 inhibit the expression of TNF- $\alpha$ by blocking the binding of LPS to CD14 cells. J Immunol 2001;167:3329-3338.

22 Gao Y, Lecker S, Post MJ, Hietaranta AJ, Li J, Volk R, Li M, Sato K, Saluja AK, Steer ML, Goldberg AL, Simons M: Inhibition of ubiquitin-proteasome pathway-mediated I kappa $B$ alpha degradation by a naturally occurring antibacterial peptide. J Clin Invest 2000;106: 439-448.

23 Bao J, Sato K, Li M, Gao Y, Abid R, Aird W, Simons M, Post MJ: PR-39 and PR-11 peptides inhibit ischemia-reperfusion injury by blocking proteasome-mediated I kappa B alpha degradation. Am J Physiol Heart Circ Physiol 2001;281:H2612-H2618. 